\title{
On Relativistic Lamé Functions
}

\author{
S. N. M. Ruijsenaars
}

\begin{abstract}
To date, the quanturn relativistic Calogero-Moser-Sutherland system with elliptic interactions is the most general one in the hierarchy of "AM-1-symmetric" integrable $M$-particle systems. We present and discuss eigenfunctions for this system. More specifically, we only deal with the $M=2$ case, but we handle a dense set in the relevant parameter space.
\end{abstract}

\section{Introduction}

The following serves to report on explicit eigenfunctions for quantum Calogero-Moser-Sutherland systems of the relativistic variety. More precisely, we restrict attontion to eigenfunctions for the case of two particles, but we do treat the most general (clliptic) type of interaction for which integrability is known to persist. Our account is based on our papers [20-22]. In keeping with these papers, we omphasize quantum-mechanical/functionalanalytic aspects. In particular, to ensure that the defining dynamics is at least formally self-adjoint, we restrict attention to real couplings and elliptic functions with a real and purely imaginary period.

We begin by recalling the Hamiltonians defining the nonrelativistic and relativistic Calogero-Moser-Sutherland systems for arbitrary particle number $M$. The nonrelativistic quantum dynamics is given by the PDO:

$$
H_{\mathrm{nr}}=-\frac{\hbar^{2}}{2 m} \sum_{j=1}^{M}\left(\frac{\partial}{\partial x_{j}}\right)^{2}+\frac{g(g-n)}{m} \sum_{\substack{j, k=1 \\ j<k}}^{M} p\left(x_{j}-x_{k}\right) .
$$

Here, $m$ is the particle mass, $g$ is the coupling constant, $h$ is Planck's constant and $\wp\left(x ; \omega, \omega^{\prime}\right)$ is the Weierstrass prfunction with half-periods $\omega$ and $\omega^{\prime}$. From now on we will set

$$
\omega=\frac{\pi}{2 r}, \omega^{\prime}=\frac{i a}{2}, \quad r, a \in(0, \infty)
$$

This somewhat unusual parametrization anticipates our conventions for the relativistic level. Here we find it convenient to work with a close relative 
of the Weierstrass o-function, viz.,

$$
s(r, a ; x)=\sigma\left(x ; \frac{\pi}{2 r}, \frac{i a}{2}\right) \exp \left(-\frac{\eta r x^{2}}{\pi}\right) .
$$

This function is cntire, odd, and $\pi / r$-antiperiodic. It satisfies the analytic difference equation

$$
\frac{s(r, a ; x+i a / 2)}{s(r, a ; x-i a / 2)}=-e^{-2 i r x},
$$

and has trigonometric and hyperbolic limits

$$
\lim _{a \rightarrow \infty} s(r, a ; x)=\frac{\sin r x}{r}, \quad \lim _{r \rightarrow 0} s(r, a ; x)=\frac{a}{\pi} \operatorname{sh}\left(\frac{\pi x}{a}\right),
$$

uriformly on compact subsets of the complex $x$-plane.

The relativistic interaction involves the functions

$$
f_{ \pm}(x)=\left(\frac{s(r, a ; x \pm i g / m c)}{s(r, a ; x)}\right)^{1 / 2}
$$

where $c$ is the speed of light; the defining quantum dynamics is then given by the analytic difference operator (henceforth $\mathrm{A} \Delta \mathrm{O}$ ):

$$
H_{\mathrm{rel}}=m c^{2} \sum_{j=1}^{M} \prod_{k \neq j} f_{-}\left(x_{j}-x_{k}\right) \exp \left(-\frac{i h \partial_{j}}{m c}\right) f_{+}\left(x_{j}-x_{k}\right)
$$

The connection between $H_{\mathrm{rel}}$ and $H_{\mathrm{n}}$ is given by the nonrelativistic limit $c \rightarrow \infty$ : One clearly gets

$$
H_{\text {rol }}=M m c^{2}+H_{\mathrm{nr}}+C_{M}+O\left(c^{-2}\right), \quad c \rightarrow \infty
$$

(Here, $C_{M}$ is a constant.) From now on, we work with the parameter $\beta=$ $1 / m c$ and put $m=\hbar=1$.

Next, we recall that the above quantum dynamics can be supplemented with $M-1$ independent and commuting $P D O$ s and $A \triangle O$ s, respectively; this is why $H_{\mathrm{nr}}$ and $H_{\text {rel }}$ are vicwed as quanturn integrable systems. Background information on the nonrelativistic systems can be found in the surveys by Olshanetsky and Perelomov [14, 15]. More recent accounts including the relativistic versions are our survey [17] and lecture notes [23].

At the quantum level there are two basic problems associated with the above formal operators. First, one wants to find joint cigenfunctions for the whole commuting family of PDOs or $A \Delta O s$, in a form that is as oxplicit as possible. Second, one wants to redefinc the opcrators as bona fide commuting self-adjoint operators on a Hilbert spacc.

We are mentioning the two problems in this order, since the second problem appears quite inaccossiblc without having explicit joint cigenfunctions 
available. More precisely, at the nonrelativistic level the Hilbert space aspects are relatively simple for $M=2$, but already hard to control for $M>2$, whereas at the relativistic level quite novel difficulties are present. In particular, it is not clear at face value that the Hamiltonian (7) can be defined as a symmetric operator on a dense subspace of the pertinent Hilbert space.

We continue by sketching the state of the art concerning the problems just mentioned, restricting attention to the elliptic settings. Beginning with the nonrelativistic level, there are explicit results only for integer $g$. For the two-particle case one is dealing with the Lamé operator

$$
H_{0}=-\frac{d^{2}}{d x^{2}}+g(g-1) \rho(x), \quad g \in \mathbb{R} .
$$

(The center-of-mass motion may be ignored.) Eigenfunctions in product form were already found by Hermite in the last century; $c f$ the last pages of Whittaker and Watson [26]. (These functions will be detailed in Section 2.) For $M>2$ the first results were obtained by Dittrich and Inozemtsev [3] (cf. also Ref. 9). More recently, Felder and Varchonko $[4,5]$ handled the arbitrary $M$, integer $g$ case in a quite different, representation-theoretic and algebro-geometric setting, obtaining eigenfunctions without addressing their quantum-mechanical features.

At the relativistic level eigenfunctions for arbitrary $M$ are only known when $g$ equals an integer, just as in the nonrelativistic case. Such eigenfunctions were quite recently constructed by Billoy [2] via a nested Bethe ansatz. See also papers by Hasegawa [8] and by Komori and Hikami [11], where it is shown (among others things) that the commuting $A \Delta O$ s admit finite-dimensional invariant subspaces spanned by theta functions. Other results relevant to the arbitrary $M$ case can be found in a recent paper by Komori [10]. He shows in particular that one can associatc a symmetric Hilbert space operator to the $A \Delta O(7)$.

Let us next specialize to the $M=2$ case. Separating off a center-of-mass factor from $H_{\text {rel }}(7)$, one winds up with the generalized Lame operator:

$$
\begin{aligned}
& H_{\beta}=\left(\frac{s(r, a ; x-i \beta g)}{s(r, a ; x)}\right)^{1 / 2} \exp \left(-i \beta \frac{d}{d x}\right)\left(\frac{s(r, a ; x+i \beta g)}{s(r, a ; x)}\right)^{1 / 2} \\
& +(i \rightarrow-i), \quad \beta>0 .
\end{aligned}
$$

We obtained integer $g$ eigenfunctions of $H_{\beta}$ in 1988, announced this in Ref. 17, and presented details in our 1994 lecture notes [23]. Integer $g$ cigenfunctions in a different guise (for $g>2$ ) were then prescnted by Krichever and Zabrodin [12], who used them to study certain solutions to their spin generalizations of the relativistic elliptic systems. Their work emphasizes the finite-gap propertics associatcd with these functions; roughly speaking, the integer $g$ equals the number of bands in the spectrum of the operators arising from (9) and (10) when one shifts $z$ over half the imaginary period. 
In this connection we also point out that a close relative $S_{0}$ of the gencralized Lamé operator $H_{\beta}$ (10) was already introduced by Sklyanin in the early eighties. He studied quite special eigenfunctions of $S_{0}$ corresponding to the band edges in the finite-gap picture $[24,25]$.

In Ref. 7 Felder and Varchenko obtained integer $g$ eigenfunctions in a form substantially equivalent to ours (cf. also Ref. 6). From their perspective, the functions arise via the algebraic Bethe ansatz, as a special case of their extensive work on representations of elliptic quanturn groups.

In our paper [20] and in the present contribution as well, we are dealing with eigenfunctions for a dense set in the parameter space $r, a, \beta>0$, $g \in \mathbb{R}$. As it turns out, these functions are in fact joint eigenfunctions of three independent commuting $A \Delta O$ s. To handle Hilbcrt space aspects, however, the spectral variable must be discretized. Thus one ends up with two commuting generalized Lamć operators, namely $H_{\beta}(10)$ and the $\mathrm{A} \Delta \mathrm{O}$ obtained by interchanging $\beta$ and $a$. (Note that from a physical point of view both $\hbar \beta$ and $a$ have dimension of length.)

It so bappens that the hyperbolic and trigonometric specializations can be treated in far more detail (cf. Refs. 21 and 22). The results obtained in these settings have their own flavor and are of independent interest. We will mostly deal with the elliptic case, however. In Section 2 we recall what is known about the two problems mentioned earlier for the integer $g$ Lamé operator $H_{0}$. We summarize these results mainly to prepare the ground for Section 3, where we consider the problems for the relativistic generalization $H_{\beta}$, choosing again $g \in \mathbb{N}$.

In Section 4 we extend the results to parameters that are dense in the natural parameter domain. To bring out some remarkable symmetry properties, we adopt another normalization and notation. In particular, this enables us to handle at once the two generalized Lamé opcrators mentioned earlicr. Section 5 contains several concluding remarks. In particular, we discuss the existence and features of interpolating eigenfunctions, and we briefly consider the hyperbolic specialization, where an explicit interpolation is known to exist $[19,23]$. We also add some speculations about $M>2$ eigenfunctions.

\section{The Nonrelativistic Integer $g$ Case}

Let us consider the time-independent Schrödinger equation

$$
-f^{\prime \prime}(x)+g(g-1) \wp(x) f(x)=E f(x),
$$

arising from the Hamiltonian $H_{0}(9)$. It is a second-order ODE to which standard existence and uniqueness results apply. Iterating the integral cquation corresponding to it, one can obtain an infinite series representation for solutions on the interval $(0, \pi / r)$ (say), with arbitrary initial conditions $f\left(x_{0}\right), f^{\prime}\left(x_{0}\right)$ for $x_{0} \in(0, \pi / r)$. 
The ODE (11) has quite special features, however. The singularity at $x=0$ is of the regular (Fuchsian) type, and the indicial equation has roots $g$ and $1-g$. For $g \notin \mathbb{Z} / 2$ one therefore obtains two fractional power series solutions with behavior $f_{1}(x) \sim x^{g}$ and $f_{2}(x) \sim x^{1-g}$ as $x \rightarrow 0$. For $g \in \mathbb{N}^{*} / 2$ there exists a power series solution behaving as $x^{g}$ for $x \rightarrow 0$, but a second linearly independent power series solution need not exist. When it exists, however (as is the case for (11) with $g \in \mathbb{N}^{*}$ ), then it behaves once more as $x^{1-g}$ for $x \rightarrow 0$.

The upshot is that in the nonrelativistic setting there is no difficulty concerning existence and uniqueness of solutions, and the solutions can actually be represented in two distinct forms. Even so, both formulas are not sufficiently explicit to get detailed information on the second problem mentioned above.

Of course, it is not a priori clear that more explicit formulas exist, but this turns out to be true for integer $g$. (These formulas can be derived in various ways; for a complete account, see the last chapter of Ref. 26.) To specify them, we put from now on

$$
g=N+1, \quad N \in \mathbb{N}^{*} .
$$

(Note that the cases $g=0,1$ are trivial.) Then the eigenfunctions of $H_{0}$ are linear combinations of functions $\mathcal{F}(x, y)$ and $\mathcal{F}(-x, y)$ of the form

$$
\mathcal{F}(x, y)=\prod_{j=1}^{N} \frac{s\left(x+z_{j}\right)}{s(x)} \cdot \exp [i r x(N+1)+i x y] .
$$

Here, $s(x)$ stands for $s(r, a ; x)$, and the spectral variable $y$ reads

$$
y=-(N+1) r+i \sum_{j=1}^{N} \frac{s^{\prime}\left(z_{j}\right)}{s\left(z_{j}\right)} .
$$

(Clearly, the first term on the right-hand side can be absorbed in $y$; it is needed for later purposes, however.) The numbers $z_{1}, \ldots, z_{N}$ ("zeros") satisfy the constraint system

$$
N \frac{s^{\prime}\left(z_{k}\right)}{s\left(z_{k}\right)}+\sum_{\substack{j=1 \\ j \neq k}}^{N} \frac{s^{\prime}\left(z_{j}-z_{k}\right)}{s\left(z_{j}-z_{k}\right)}-\sum_{j=1}^{N} \frac{s^{\prime}\left(z_{j}\right)}{s\left(z_{j}\right)}=0, \quad k=1_{1} \ldots, N
$$

and this system admits a solution curve. (Note that the sum of the $N$ lefthand-sides vanishes identically, so that this is a priori plausible.) Adding the rclation (14) between $y$ and $z_{1}, \ldots, z_{N}$ to the system, one may view $y$ as the curve parameter. For $y \in(K, \infty)$ with $K$ sufficiently large, one can then choose $z_{j}=i \epsilon_{j}(y), \epsilon_{j}>0$, with $\epsilon_{j} \downarrow 0$ for $y \uparrow \infty$. 
It is not obvious, but true that $F(x, y)$ thus definod is a solution to (11) with $E$ given by

$$
E=-(2 N-1) \sum_{j=1}^{N} \wp\left(z_{j}\right)
$$

Thus one gets $E=E(y) \uparrow \infty$ as $y \uparrow \infty$.

The functions $\mathcal{F}( \pm x, y)$ clearly become singular as $x^{-N}=x^{1-9}$ for $x \rightarrow$ 0 , in agreement with Fuchs thcory. Morcover, the functions

$$
\Phi(x, y) \equiv \mathcal{F}(x, y)-(-1)^{N} \mathcal{F}(-x, y)
$$

vanish as $x^{N+1}$ for $x \perp 0$. (Again this is not obvious at first sight. Note, however, that the leading singularity $x^{-N}$ is taken out in $\Phi$, so that its $x^{N+1}$-behavior follows from Fuchs theory.)

Next, note that $\mathcal{F}(x, y)$ satisfies the quasi-periodicity relation

$$
\mathcal{F}\left(x+\frac{\pi}{r}, y\right)=(-1)^{N+1} \exp \left(\frac{i \pi y}{r}\right) \mathcal{F}(x, y)
$$

Hence $\mathcal{F}(x, n r), n \in \mathbb{N}$, is $\pi / r$-periodic or -antiperiodic. As a consequence, the functions

$$
\Phi_{n}(x) \equiv \Phi(x, n r), \quad n \in \mathbb{N},
$$

vanish not only at $x=0$, but also at $x=\pi / r$. Thus they belong to the Hilbert space

$$
\mathcal{H} \equiv L^{2}((0, \pi / r), d x)
$$

of square-integrable functions on the interval $(0, \pi / r)$. For $n \rightarrow \infty$ one gets $E_{n}=E(n r) \uparrow \infty$, so the eigenvalues of $H_{0}$ on $\Phi_{n}$ are distinct for $n$ large. It now follows from a standard argument that the functions $\Phi_{n}$ are pairwise orthogonal for $n$ large.

More generally, from the woll-developed self-adjointness theory for ordinary differential operators one readily deduces that $H_{0}$ is essentially selfadjoint on $C_{0}^{\infty}((0, \pi / r))$ for $g \geq 3 / 2$. Moreover, the Weyl-Kodaira-Titchmarsh theory of eigenfunction expansions yiclds the existence of an orthonormal base of eigenfunctions. It is natural to expect that the latter is given by the (renormalized) functions $\left\{\Phi_{n}\right\}_{n=0}^{\infty}$, but to our knowledge this has not even been shown for $g=2$.

In this connection it should be noted that the $g=2$ case is particularly accessible, since the constraint system (15) is trivial for $N=1$. From (14) one then sces that the sequence of values $y=0, r, 2 r, \ldots$ yiclds a sequence of distinct $z_{1}$-values $z_{1}(n r)$ in the interval $i(0, a / 2)$. The corresponding energies $E_{n}$ are obviously distinct, too (cf. (16)), so the functions $\Phi_{0}, \Phi_{1}$, 
... are well-defined, nonzero, and pairwise orthogonal. (But it is not clear that they are complete.)

By contrast, the system (15) for $N>1$ is quite inaccessible. Though results for generic $y \in \mathbb{C}$ can be gleaned from Refs. 26 and 5 , the Hilbert space aspects involvo the (nongeneric) values $y=n r$. (It is not clear, for example whether the functions $\Phi_{n}$ are nonzero for $n$ small and whether the eigenvalues $E_{n}$ are distinct.) Thus, even in the nonrelativistic integer $g$ contcxt the Hilbert space questions have not been completely elucidated.

On the other hand, for the trigonometric specialization we have

$$
\lim _{a \rightarrow \infty} \wp\left(x ; \frac{\pi}{2 r}, \frac{i a}{2}\right)=\frac{r^{2}}{\sin ^{2}(r x)}-\frac{r^{2}}{3} .
$$

For this potential the Hilbert space theory is in great shape. Indeed, in the trigonometric case the above functions $\Phi_{n}(x)$ and eigenvalues $E_{n}$ can be seen to be of the form

$$
\begin{aligned}
\Phi_{n}(x) & =w(x)^{1 / 2} P_{n}(\cos r x), \quad n \in \mathbb{N}, \\
E_{n} & =(n+N+1)^{2} r^{2}-(N+1) N \frac{r^{2}}{3} .
\end{aligned}
$$

Here, the functions $P_{n}(u)$ are Gegenbauer polynomials, of degree $n$ and parity $(-1)^{n}$, and

$$
w(x)=(\sin r x)^{2 N+2}
$$

amounts to the weight function with regard to which they are orthogonal. Therefore, completeness is obvious, and this is one important reason to conjecture that for $a<\infty$ the functions $\Phi_{0}, \Phi_{1}, \ldots$ are still complete. Unfortunately, no representation analogous to (22) is known for the elliptic case.

\section{The Relativistic Integer $g$ Case}

Let us now turn to a consideration of the $\mathrm{A} \Delta \mathrm{O} H_{\beta}(10)$. Here the timeindependent Schrödinger equation is an analytic difference equation ( $A \triangle E$ ), viz.,

$$
\left(\frac{s(x-i \beta g)}{s(x)} \cdot \frac{s(x-i \beta+i \beta g)}{s(x-i \beta)}\right)^{1 / 2} F(x-i \beta)+(i \rightarrow-i)=E F(x) .
$$

The theory of such equations is far less developed than for ordinary differential and discrete difference equations. Though some existence results are known, the main problem is to single out solutions with special properties. Indeed, the kcy difference between the $\mathrm{ODE}$ (11) and the $\mathrm{A} \triangle \mathrm{E}(25)$ is that 
the former has a two-dimensional solution space, whereas for the latter the existence of onc nontrivial solution $F_{E}(x)$ already entails that the solution space is infinite-dimensional: For any meromorphic (say) multiplier $M(x)$ with period $i \beta$ the function $M(x) F_{E}(x)$ solves (25) as well.

The problem is, then, to find solutions with special properties, preferably such that they can be used to define the $A \Delta O H_{\beta}(10)$ as a genuine selfadjoint operator on $\mathcal{H}(20)$. The point is that there is no obvious way to define $H_{\beta}$ first as a symmetric operator on a dense subspace, by contrast to $H_{0}$, where for instance $C_{0}^{\infty}((0, \pi / r))$ serves this purpose. Since no general Hilbert space theory for $A \Delta O$ s exists at the present time, one may instead try to find sufficiently explicit pairwise orthogonal eigenfunctions $\Phi_{n} \in \mathcal{H}$ with real eigenvalues $E_{n}$. Setting then $H \Phi_{n} \equiv E_{n} \Phi_{n}$, extending linearly, and taking the Hilbert space closure $\bar{H}$ of the symmetric operator $H$ thus defined, one obtains a self-adjoint operator $\vec{H}$ on (a dense subspace of) the closed subspace spanned by the pertinent eigenfunctions.

As it turns out, this scenario can be realized to a large extcnt. We continue by describing the eigenfunctions that generalize the above eigenfunctions $\mathcal{F}(x, y)$ and that play the desired role in rigorously redefining $H_{\beta}$ as a self-adjoint quantum dynamics. Choosing as before $g=N+1$ with $N \in \mathbb{N}^{*}$, and requiring first

$$
2 N \beta \in(0, a),
$$

they are of the form

$$
\mathcal{F}(x, y)=\prod_{j=1}^{N} \frac{s\left(x+z_{j}\right)}{[s(x+i j \beta) s(x-i j \beta)]^{1 / 2}} \cdot \exp [i r x(N+1)+i x y] .
$$

Here, the spectral variable $y$ is related to the zcro functions via

$$
y=-(N+1) r-\frac{1}{2 \beta} \ln \left(\prod_{j=1}^{N} \frac{s\left(z_{j}-i \beta\right)}{s\left(z_{j}+i \beta\right)}\right)
$$

and the latter obey the constraint system

$$
\begin{array}{r}
s\left(z_{k}-i N \beta\right) \prod_{j \neq k} s\left(z_{j}-z_{k}-i \beta\right) \prod_{j} s\left(z_{j}+i \beta\right)-(\beta \rightarrow-\beta)=0, \\
k=1, \ldots, N .
\end{array}
$$

It is clear that for $\beta \downarrow 0$ these equations yicld the nonrelativistic counterparts (13)-(15). But in contrast to (15), it is by na means clcar that one of the $N$ equations for the $N$ unknowns $z_{1}, \ldots, z_{N}$ is a consequence of the remaining $N-1$ equations. This is, however, truc, and it is important to understand the reason. Viewing (27) as an Ansatz for solving (25) with 
$g=N+1$, one obtains the function

$$
\begin{aligned}
& E \equiv \frac{1}{s(x)}\left(s(x+i N \beta) \exp [(N+1) \beta r+\beta y] \prod_{j=1}^{N} \frac{s\left(x-i \beta+z_{j}\right)}{s\left(x+z_{j}\right)}\right. \\
& +(\beta \rightarrow-\beta)) \text {. }
\end{aligned}
$$

Of course, this function depends on $x$ when one gives $z_{1}, \ldots, z_{N}$ arbitrary values. But since the function is elliptic in $x$ (with periods $\pi / r, i a$, cf. (4)), one need only require that the residues at $N$ of its $N+1$ (generically) simple poles in a period cell vanish to ensure that it is constant. Now the requirement that the residue at $x=0$ vanish yields (28), whereas the residues at $x=-z_{k}$ give rise to (29). Thus we need only prove that the system (29) with $k=2, \ldots, N$ (say) admits a solution curve to infer that all of the equations are solved.

Now it is obvious that all of the $N$ equations are solved by choosing

$$
z_{j}=i j \beta, \quad j=1, \ldots, N \text {. }
$$

An application of the implicit function theorem then shows that the equations with $k=2, \ldots, N$ have a unique holomorphic solution $z_{k}\left(z_{1}\right), k=2$, $\ldots, N$, near (31). Moreover, taking $z_{1}(t)=i \beta+i t$ with $t \in[0, \epsilon)$, the functions $z_{j}\left(z_{1}(t)\right)$ are real-analytic functions from $[0, \epsilon)$ to $i(0, \infty)$ for $\epsilon$ small enough. From (28) it is then clear that (eventually decreasing $\epsilon$ ) $y=y(t)$ is real-analytic and real-valued on $(0, \epsilon)$, and that one has $y \uparrow \infty$ for $t \downarrow 0$.

As a consequence, one can trade $t$ for $y$ in a neighborhood $(K, \infty)$ of $\infty$. Since we know very littlc about the minimal $K$ satisfying various requirements, we may increase $K$ as the need arises. In particular, we can choose it sufficiently large so that the functions

$$
\Phi_{n}(x) \equiv \mathcal{F}(x, n r)-\mathcal{F}(-x, n r), \quad n r>K, n \in \mathbb{N},
$$

arc well defined and nonzero. Indeed, the above functions $z_{j}\left(z_{1}(t(y))\right)$ (denoted simply $z_{j}(y)$ from now on) satisfy

$$
y \uparrow \infty \Longrightarrow z_{j}(y) \rightarrow i j \beta, j=1, \ldots, N,
$$

so that the summands on the right-hand side of (32) have distinct zeros for $y$ large enough. (Recall our standing assumption (26).) Moreover, taking $x=i N \beta$ in (30) (which we may do, since $E$ is $x$-independent), one deduces that an eventual increase of $K$ ensures $E(y)$ is increasing on $(K, \infty)$. Then $\mathrm{H}_{\beta}$ has distinct eigenvalues on $(K, \infty)$.

The crux is now that all of the functions $\Phi_{n}(x)$ just defined belong to a dense subspace $\mathcal{A} \subset \mathcal{H}$ such that $H_{\beta} \mathcal{A} \subset \mathcal{H}$ and such that $H_{\beta}$ is symmetric on $\mathcal{A}$. It is important to point out that the definition of $\mathcal{A}$ (which we do not present herc) is not directly motivated by $H_{\beta}$, but rather by properties of 
the above (very speciall) $H_{a}$-cigenfunctions. Since the eigenvalues $E_{n}$ and $E_{m}$ are distinct for $n \neq m$, it now follows from symmetry that $\Phi_{n}$ and $\Phi_{m}$ are orthogonal. Thus we obtain a self adjoint operator (denoted again $H_{\beta}$ ) on the closed subspace $\mathcal{H}_{K} \subset \mathcal{H}$ spanned by the functions (32).

We expect that $\mathcal{H}_{K}$ equals $\mathcal{H}$ whenever $K$ can be chosen ncgative. Put differently, we conjecture that for $K<0$ the functions $\Phi_{0}, \Phi_{1}, \ldots$ are an orthogonal base for $\mathcal{H}$. In the special case $N=1$ one can choose $K=$ $-r$ (cf. Ref. 22, Eq. (2.35)), but completeness is still open, even in this simple case. More generally, we expect that the orthocomplement of $\mathcal{H}_{K}$ is spanned by functions $\Phi_{0}, \ldots, \Phi_{[K / r]}$ that are eigenfunctions of $H_{j}$ with real eigenvalues.

Once again, the orthogonality and completeness problems are trivial for the trigonometric specialization, since one winds up with orthogonal polynomials in that case. Specifically, for $a=\infty$ the functions $\Phi_{n}$ are still of the form (22), with the weight function (24) now given by

$$
w(x)=\sin ^{2}(r x) \prod_{j=1}^{N} \sin r(x-i j \beta) \sin r(x+i j \beta) .
$$

The associated orthogonal polynomiais are then $q$-Gegenbauer polynomials (cf., for example, Ref. 1), with $q$ given by

$$
q=\exp (-2 \beta r) \text {. }
$$

Returning to the elliptic case, we recall that we have restricted $\beta$ by (26) in the above account. But a substantial part of our results continues to be valid under the restriction

$$
k \beta \notin \mathbb{N} a, \quad k=1, \ldots, 2 N .
$$

In particular, this suffices to infer the existence of cigenfunctions of the form $(27)-(29)$. (Note that this more general restriction still guarantees that for $y$ large the zeros $z_{1}(y), \ldots, z_{N}(y),-z_{1}(y), \ldots,-z_{N}(y)$ are distinct modulo the period ia, of. (33).)

A key difference is, however, that for $N \beta>a$ the functions $\Phi_{n}(32)$ are most likely no longer pairwise orthogonal. More precisely, our symmetry prouf breaks down for $N \beta>a$, and orthogonality is indeed violated in all cases where this could be tested. For $N=1$ and $\beta>a$ a breakdown of orthogonality occurs in the strongest possible form: Onc has $\left(\Phi_{n}, \Phi_{m}\right) \neq 0$ for all $n \neq m$. (Here, we still assume (36); note that $H_{\beta}$ becomes "free" for $N=1$ and $\beta=l a / 2, i \in \mathbb{N}^{*}$.)

We prove the latter assertion in Rel. 22, which is concerned with the $g=2$ case. To conclude this section we mention another remarkable result from this paper. Taking $\beta \uparrow a$ (the edge of the unitarity region) and simultaneously $a \backslash 0$ in a certain way, the above cigenfunctions $\Phi_{n}(x)$ converge to the Lieb-Liniger eigenfunetions $[13]$ for the $(M=2$, center-of-mass $)$ 
repulsive delta-function Bose gas. The role of the finite volume in [13] is played by the elliptic period $\pi / r<\infty$. This limiting transition generalizes the connection between the $g=2$ hyperbolic relativistic $M=2$ eigenfunctions and the infinate-volume delta-function eigenfunctions, which wo pointed out at the end of [17].

\section{Eigenfunctions for a Dense Parameter Set}

Thus far, we have viewed the functions $\mathcal{F}(x, y)(27)$ as eigenfunctions of $H_{\text {ig }}$ (10). However, they also satisfy the quasi-periodicity rclations (cf. (4))

$$
\begin{aligned}
& \mathcal{F}(x+i a, y)=\exp \left[-2 u r \sum_{j=1}^{N} z_{j}(y)-(N+1) a r-a y\right] \mathcal{F}(x, y), \\
& \mathcal{F}\left(x+\frac{\pi}{r}, y\right)=-\exp \left(\frac{i \pi y}{r}\right) \mathcal{F}(x, y) .
\end{aligned}
$$

Thus, they can also be regarded as eigenfunctions of the $A \Delta O s T_{ \pm i a}$ and $T_{ \pm \pi / \text {, }}$ where we use the notation

$$
\left(T_{\alpha} F\right)(x) \equiv F(x-\alpha), \quad \alpha \in \mathbb{C} .
$$

Now this is true for $\mathcal{F}(-x, y)$, too, but then we obtain different eigenvalues. On the other hand, introducing the "extra" $A \Delta O$,

$$
H_{e} \equiv T_{i a}+T_{-i a}
$$

and the "quasi-periodicity" $A \Delta O$,

$$
Q=T_{\pi / r}+T_{-\pi / r}
$$

we obtain the same eigenvalues for $\mathcal{F}(x, y)$ and $\mathcal{F}(-x, y)$. Hence the functions $\mathcal{F}( \pm x, y)$ are joint cigenfunctions of the triple of independent $A \Delta O s$ $\left(H_{\beta}, H_{e}, Q\right)$.

As will now be detailed, we have found eigenfunctions $\mathcal{F}( \pm x, y)$ of $H_{\beta}$ for a set that is dense in the parameter space $r, \beta, a>0, g \in \mathbb{R}$. These eigenfunctions of $H_{\beta}$ are once again cigenfunctions of $Q$ with eigenvalue $-2 \cos (\pi y / r)$ and of an extra $A \Delta O H_{e}$ involving the shifts $T_{ \pm i a}$. But the latter operator is no longer "free": It involves the functions (6) with an interchange of $a$ and $\hbar \beta=\hbar / m c$. Within this more general setting, $H_{\beta}$ and $H_{c}$ arc on the same footing from a mathematical viewpoint, and we take this into account by switching to notation that makes this symmetry manifest.

Spceifically, we work from now on with parameters $a_{+}, a_{-}$, and $b$ defined by

$$
a_{+}=\beta, \quad a_{-.}=a, \quad b=\beta g .
$$


The pertinent parameter domain at the elliptic level is then

$$
\varepsilon \equiv\left\{\left(r, a_{+}, a_{-}, b\right) \in(0, \infty)^{3} \times \mathbb{R}\right\} .
$$

Introducing the notation

$$
s_{\delta}(x) \equiv s\left(r, a_{\delta} ; x\right), \quad \delta=+,-,
$$

we now define the $A \Delta O$ s

$$
\begin{array}{r}
H_{b} \equiv e^{-b r}\left(\left(\frac{s_{\delta}(x-i b)}{s_{b}(x)}\right)^{1 / 2} T_{i a_{-\alpha}}\left(\frac{s_{\delta}(x+i b)}{s_{b}(x)}\right)^{1 / 2}+(i \rightarrow-i)\right), \\
\delta=+,-.
\end{array}
$$

Comparing $H_{\beta}(10)$, one secs that $H_{-}$arises from $H_{\beta}$ via the substitutions (42), but for the prefactor $\exp (-b r)$. Likewise, taking $g=N+1$, the $\mathrm{A} \Delta \mathrm{O}$ $H_{+}$reduces to a positive multiple of $H_{e}(40)$. The choice of prefactor in (45) ensures that the operators thus defined satisfy the invariance property

$$
H_{\delta}\left(a_{+}+a_{m}-b\right)=H_{\delta}(b), \quad \delta=+,-.
$$

The assertions just made can be easily verified by using the $A \triangle E$ (4). Similarly, this $\mathrm{A} \Delta \mathrm{E}$ can be used to check that $H_{+}$and $H_{-}$commute. Now this is in accord with the existence of joint eigenfunctions, but there are no general results to the effect that commutativity of two A $\Delta O \mathrm{O}$ implies the existence of joint eigenfunctions. (In this connection it is important to observe that when the two summands of $H_{+}$are multiplied by meromorphic functions with period $i a_{+}$, the resulting $\mathrm{A} \Delta \mathrm{O}$ still commutes with $H_{-}$.)

Even so, we have found joint eigenfunctions $\mathcal{F}( \pm x, y)$ of the three independent commuting $\mathrm{A} \Delta \mathrm{O} \otimes H_{+}, H_{-}$and $Q$ for a dense set $\mathcal{D}$ in $\mathcal{E}$ (43). For expository simplicity, we will specify these functions for a subset $\mathcal{D}_{+} \cup D_{-}$ of $D$ that is already dense. The two sets $\mathcal{D}_{\alpha}, \alpha \in\{+,-\}$, are defined by

$$
\begin{array}{r}
\mathcal{D}_{\alpha} \equiv\left\{\left(r, a_{+}, a_{-}, b\right) \in \mathcal{E} \mid b=\left(N_{\alpha}+1\right) a_{\alpha}-N_{-\alpha} a_{-\alpha},\right. \\
\left.N_{+}, N_{-} \in \mathbb{N}, a_{+} / a_{-} \notin \mathbb{Q}\right\} .
\end{array}
$$

Since the quotient $a_{+} / a_{-}$is allowed to be an arbitrary positive irrational number, the $b$-values occurring here are dense in $\mathbb{R}$. Hence each of the two (disjoint) sets $\mathcal{D}_{+}$and $\mathcal{D}_{-}$is dense in $\mathcal{E}$. To visualize the situation, it may be helpful to inspect Figure 26.1, where we have fixed $a_{-}$and drawn some of the pertinent lines in the $\left(a_{+}, b\right)$-plane.

The two sets $\mathcal{D}_{+}, \mathcal{D}_{-}$are interchanged under the transformation $b \rightarrow$ $a_{+}+a_{-}-b$. Fixing $r>0, a_{+} / a_{-} \notin \mathbb{Q}$ and $N_{+}, N_{-} \in \mathbb{N}$, we get a point in $\mathcal{D}_{+}$by taking $b=\left(N_{+}+1\right) a_{+}-N_{-} a_{-}$and a point in $\mathcal{D}$ - by taking 

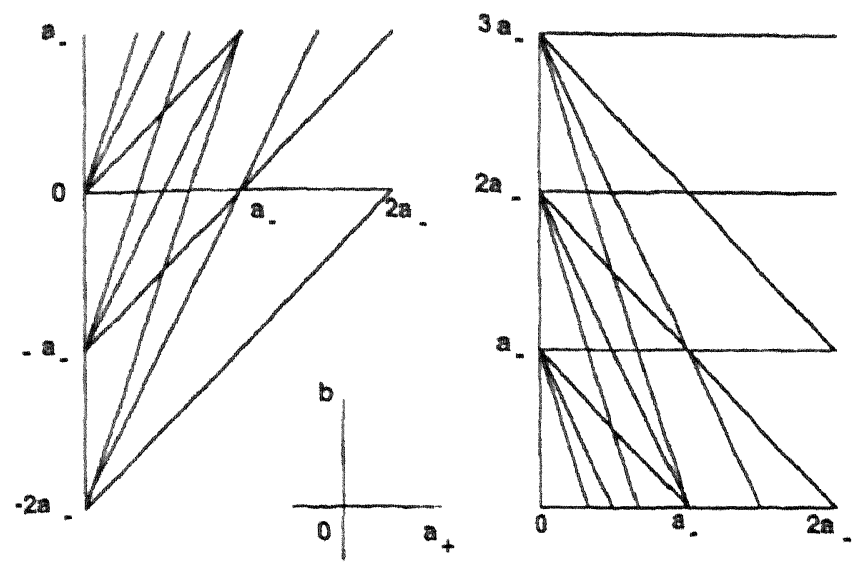

FIGURE 26.1. Some lines in the $\left(a_{+}, b\right)$-plane belonging to $D_{+}$(left) and $D_{-}$ (right); the parameter $a_{-}$is fixed.

$b=\left(N_{-}+1\right) a_{-}-N_{+} a_{+}$. In agreement with the invariance property (46), the joint eigenfunctions $\mathcal{F}( \pm x, y)$ are the same in both points. They read

$$
\begin{aligned}
\mathcal{F}(x, y)=\prod_{\delta=+,-} \prod_{j=1}^{N_{\delta}} \frac{s_{-\delta}\left(x+z_{j}^{\delta}(y)\right)}{\left[s_{-\delta}\left(x+i j a_{\delta}\right) s_{-\delta}\left(x-i j a_{\delta}\right)\right]^{1 / 2}} \\
\cdot \exp \left[i r x\left(2 N_{+} N_{-}+N_{+}+N_{-}+1\right)+i x y\right] .
\end{aligned}
$$

The zero functions $z_{j}^{\delta}, j=1, \ldots, N_{\delta}$, satisfy the constraint system (29) with $N \rightarrow N_{\delta}, \delta=+,-$, and the two systems are coupled via the spectral variable $y$ in a somewhat involved way that we will not detail here.

We do specify the asymptotics of the zero functions and eigenvalues:

$$
\begin{aligned}
& y \uparrow \infty \Longrightarrow z_{j}^{\delta}(y) \rightarrow i j a_{\delta}, \quad j=1, \ldots, N_{\delta}, \delta=+,-, \\
& y \uparrow \infty \Longrightarrow E_{\delta}(y) \sim \exp \left(a_{-\delta} y\right), \frac{d E_{\delta}(y)}{d y} \sim a_{-\delta} \exp \left(a_{-\delta} y\right), \\
& \delta=+,-.
\end{aligned}
$$

In view of the eigenvalue asymptotics, we can choose $K$ such that on $(K, \infty)$ the two eigenvalues separate points:

$$
K<y_{1}<y_{2} \Longrightarrow\left(E_{+}\left(y_{1}\right), E_{-}\left(y_{1}\right)\right) \neq\left(E_{+}\left(y_{2}\right), E_{-}\left(y_{2}\right)\right) .
$$

Another important feature is that the joint eigenspace is two-dimensional. (Again, we can only prove this for $y \in(L, \infty)$ and sufficiently large $L \geq$ $K$, cf. Appendix B in Ref. 20.) Notice that this is false when $a_{+} / a_{-}$is 
rational: In that case joint eigenspaces are infinite-dimensional whenever they contain one nontrivial function.

We now turn to Hilbert space aspects. To this end we study the functions (32), with $f$ now given by (48). These functions are clearly $\pi / r$-periodic (-antiperiodic) for $n$ odd (even). It is far from clear, but true that they are also pair wise orthogonal in $\mathcal{H}(20)$, provided $b$ belongs to the unitarity interval $\left(0, a_{+}+a_{-}\right.$. Fixing once more $a_{-}$, we have depicted the resulting unitarity region in the $\left(a_{+}, b\right)$-plane in Figure 26.2.

As a consequence, $H_{+}$and $H_{-}$can be redefined as commuting self-adjoint operators on the closed subspace $\mathcal{H}_{K}$ spanned by the functions (32): We need unly set

$$
H_{\delta} \Phi_{n} \equiv E_{b}(n r) \Phi_{n}, \quad n r>K, n \in \mathbb{N}
$$

extend linearly, and take the closure.

Just as in the special case $N_{-}=0$ (cf. Section 3), orthogonality is most likely violated for $b<0$ and $b>a_{+}+a_{-}$. We also expect that for $b \in$ $\left(0, a_{+}+a_{-}\right)$the subspace $\mathcal{H}_{K}^{\frac{1}{K}}$ has dimension $[K / r]+1$ and is spanned by joint eigenfunctions $\Phi_{0}, \ldots, \Phi_{[K / n]}$ with real eigenvalues.

We conclude this section by explaining a key feature in the symmetry/orthogonality analysis. To this end we first rewrite $\mathcal{F}(x, y)$ as

$$
\mathcal{F}(x, y)=\mathcal{H}(x, y) / \prod_{\delta=+-} \prod_{j=1}^{N_{\delta}}\left[s_{-\delta}\left(x+i j a_{\delta}\right) s_{-\delta}\left(x-i j a_{\delta}\right)\right]^{1 / 2},
$$

so that we have

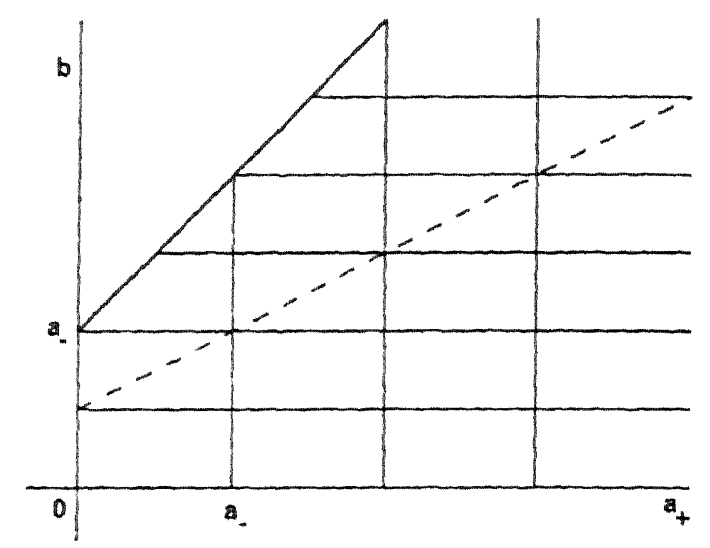

FIGURE 26.2. The unitarity region and the symmetry line $b=\left(a_{+}+a_{-}\right) / 2$ (dashed) in the $\left(a_{+}, b\right)$-plane 


$$
\mathcal{H}(x, y)=\prod_{\delta=+,-} \prod_{j=1}^{N_{\delta}} s_{-\delta}\left(x+z_{j}^{\delta}(y)\right)
$$

Note that this similarity transformation yields holomorphic eigenfunctions. By exploiting the transformed A $\triangle E s$ it can now be proved that one has the identities

$$
\begin{aligned}
\mathcal{H}\left(i k_{+} a_{+}+i k_{-} a_{-}, y\right)= & \mathcal{H}\left(-i k_{+} a_{+}-i k_{-} a_{-}, y\right) \\
& k_{\delta} \in\left\{-N_{\delta}, \ldots, 0, \ldots, N_{\delta}\right\}, \delta=+,-.
\end{aligned}
$$

Therefore, the function $\mathcal{H}(x, y)-\mathcal{H}(-x, y)$ has $\left(2 N_{+}+1\right)\left(2 N_{-}+1\right)$ explicitly known zeros on the imaginary axis. Since the functions

$$
\psi_{n}(x) \equiv \mathcal{H}(x, n r)-\mathcal{H}(-x, n r)
$$

are $\pi / r$-periodic ( $n$ odd) or $\pi / r$-antiperiodic ( $n$ even), the above zeros repeat under a shift by $\pi / r$ :

$$
\begin{aligned}
\psi_{n}\left(i k_{+} a_{+}+i k_{-} a_{-}+\frac{l \pi}{r}\right) & =0, \\
& k_{\delta} \in\left\{-N_{\delta}, \ldots, 0, \ldots, N_{\delta}\right\}, \delta=+,-, l \in \mathbb{Z} .
\end{aligned}
$$

These explicit zeros of $\psi_{n}(x)$ are crucial in canceling poles arising from the squared denominator in (48). But only for $b \in\left(0, a_{+}+a_{-}\right)$one gets a pole-zero cancellation in a strip around the real $x$-axis that suffices to deduce pairwise orthogonality. For $b<0$ and $b>a_{+}+a_{-}$one obtains instead a residue sum that has no reason to vanish (although in general its vanishing cannot be ruled out).

\section{Concluding Remarks}

In this final section we sketch some more results related to the above eigenfunctions. First, we point out that the functions $\mathcal{F}( \pm x, y)(48)$ can also be viewed as joint eigenfunctions of $H_{+}$and $H_{-}$for the $b$-values $-N_{+} a_{+}-N_{-} a_{-}$and $\left(N_{+}+1\right) a_{+}+\left(N_{-}+1\right) a_{-}$(in addition to $b=$ $\left.\left(N_{\alpha}+1\right) a_{\alpha}-N_{-\alpha} a_{-\alpha}, \alpha=+,-\right)$. This is because one has the identities

$$
\begin{aligned}
H_{\delta}\left(-N_{+} a_{+}-N_{-} a_{-}\right) & =H_{\delta}\left(\left(N_{+}+1\right) a_{+}+\left(N_{-}+1\right) a_{-}\right) \\
& =r_{\delta} H_{\delta}\left(\left(N_{+}+1\right) a_{+}-N_{-} a_{-}\right)
\end{aligned}
$$

where 


$$
r_{b} \equiv \exp \left(2 N_{+}+1\right)\left(2 N_{-}+1\right) a_{-} \delta r .
$$

(The first equality follows from (46); the sccond can be checked using (4).) To obtain once again the eigenvalue asymptotics (50) for these new bvalues, one should, however, shift the spectral variable $y$ over a distance $\left(2 N_{+}+1\right)\left(2 N_{-}+1\right) r$.

More generally, since we can handle a dense subset of $\mathcal{E}$ (43), a natural question concerns the existence of continuous interpolating joint eigenfunctions for all of $\mathcal{E}$. Clearly, such interpolations are uniquely determined up to multipliers depending solely on $y$ and the parameters. Now a crucial feature of the above functions $\mathcal{F}(x, y)$ is that their $y \rightarrow \infty$ asymptotics is given in terms of a scattering function $u\left(r, a_{+}, a_{-}, b ; x\right)$ that has a real-analytic extension to all of $\mathcal{E}$ (taking $x \in \mathbb{R}$ ), as will now be detailed.

First, we note that (49) entails

$$
\mathcal{F}(x, y) \sim \zeta\left(N_{+}, N_{-}\right)[-\exp (2 i r x) u(x)]^{1 / 2} \operatorname{cxp}(i x y), \quad y \rightarrow \infty,
$$

where $\zeta$ is a suitable phase and where

$$
\begin{aligned}
u(x)=(-1)^{N_{+}+N_{-}} \prod_{\delta=+-} \prod_{j=1}^{N_{\delta}} \frac{s_{\delta}\left(x+i j a_{-} \delta\right)}{s_{\delta}\left(x-i j a_{-}\right)} \\
\quad x \exp \left[2 i r x\left(2 N_{+} N_{-}+N_{+}+N_{-}\right)\right] .
\end{aligned}
$$

To explain why this function extends to all of $\mathcal{E}$ (and for other purposes), it is convenient to introduce a function

$$
c\left(r, a_{+}, a_{-}, b ; x\right)=\frac{G\left(r, a_{+}, a_{-} ; x-i b+i\left(a_{+}+a_{--}\right) / 2\right)}{G\left(r, a_{+}, a_{-} ; x+i\left(a_{+}+a_{-}\right) / 2\right)} .
$$

Here, $G\left(r, a_{+}, a_{-} ; z\right)$ is the elliptic generalized gamma function introduced and studied in Ref. 18. It is meromorphic in $z$ and in the parameters $r, a_{+}$ and $a_{-}$as long as $r a_{+}$and $r a_{-}$stay in the right half-plane. Now the extension to $\mathcal{E}$ of the function $u(x)=u\left(r, a_{+}, a_{-},\left(N_{\alpha}+1\right) a_{\alpha}-N_{-\alpha} a_{-\alpha} ; x\right)$ given by (61) reads

$$
u(x)=-e^{-2 i r x} \frac{c(x)}{c(-x)} .
$$

For real $x$ this function is real-analytic on $\mathcal{E}$, as advertized. In particular, it is uniquely determined by (61). Thus the asymptotics of the joint eigenfunctions admits a unique real-analytic interpolation.

The similarity transformation (53) turning the two-valued cigenfunction $\mathcal{F}(x, y)$ into the holomorphic function $\mathcal{H}(x, y)$ is readily scen not to admit a continuous interpolation. But when we introduce a generalized weight function

$$
w(x)=\frac{1}{c(x) c(-x)}
$$


then the similarity transformation to a new, meromorphic function

$$
\Psi(x, y) \equiv \frac{\mathcal{F}(x, y)}{\zeta\left(N_{+}, N_{-}\right) w(x)^{1 / 2}},
$$

does admit an interpolation, since $w(x)$ does. The function $\Psi$ has asymptotics

$$
\Psi(x, y) \sim c(x) e^{i \Sigma \eta}, \quad y \rightarrow \infty,
$$

and correspondingly $c(x)$ may be viewed as a generalization of the HarishChandra c-function for symmetric spaces of rank 1 (cf. Refs. 15 and 17).

Of course, this does not answer the question of whether the meromorphic joint eigenfunctions $\Psi( \pm x, y)$ of the similarity-transformed $A \Delta O$ s

$$
\begin{aligned}
& A_{b} \equiv w(x)^{-1 / 2} H_{b} w(x)^{1 / 2}=e^{-b r} \frac{s_{\delta}(x-i b)}{s_{\delta}(x)} T_{i a_{-b}}+(i \rightarrow-i), \\
& \delta=+,-,
\end{aligned}
$$

admit an extension to $\mathcal{E}$. This appears to be a quitc delicate issue. For the even combination

$$
\chi(x, y) \equiv \Psi(x, y)+\Psi(-x, y)
$$

the identities (55) give rise to pole-zero cancellations on the imaginary axis, but as before one needs to choose $y=n r, n \in \mathbb{N}$, to ensure the same cancellation on the lines $\operatorname{Re} x=k \pi / r, k \in \mathbb{Z}^{*}$.

These cancellations are not only crucial for the orthogonality issue, but they are also relevant for the question of meromorphic intorpolations. Indeed, for convergence to points in $\mathcal{E}$ for which $a_{+} / a_{-}$is irrational and $b$ not equal to $k a_{+}+l a_{-}$with $k, l$ integers (for example), one needs to let $N_{+}$and $N_{-}$go to $\infty$; hence cancellations are needed to prevent poles from becoming dense.

Even so, it appears hard to exclude the existence of a meromorphic interpolation for $\Psi(x, y)$. (This is because poles of meromorphic functions can exhibit drastic changes under convergence.) At any rate, for Hilbert space purposes it would suffice to control the convergence to arbitrary points in $\mathcal{E}$ for the functions $\chi(x, n r), n \in \mathbb{N}$.

Though we have discussed these questions in more detail in Ref. 20 , we have obtained no clear-cut answers. By contrast, at the hyperbolic level an interpolation of $\chi(x, y)$ is explicitly known. More precisely, there exists a function $R\left(a_{+}, a_{-}, b ; x, p\right)$ that is real-analytic in $a_{+}, a_{-}$, and $b$ in the hyperbolic parameter dornain $\left\{a_{+}, a_{-}>0, b \in \mathbb{R}\right\}$ for $x, p$ fixed, meromorphic in $x, p$ for fixed parameters, and that satisfies

$$
\begin{aligned}
& R\left(a_{+}, a_{-}, k a_{+}+l a_{-} ; x, p\right) \\
& \quad=r_{k l}\left(a_{+}, a_{-} ; p\right) \times\left(a_{+}, a_{-}, k a_{+}+l a_{-} ; x, \pi p / a_{+} a_{-}\right), \quad k, l \in \mathbb{Z} .
\end{aligned}
$$


The renormalization of the dependence on the spectral variable ensures the self-duality property

$$
R\left(a_{+}, a_{-}, b ; x, p\right)=R\left(a_{+}, a_{-}, b ; p, x\right) .
$$

Furthermore, one has the symmetry property

$$
R\left(a_{+}, a_{-}, b ; x, p\right)=R\left(a_{-}, a_{+}, b ; x, p\right),
$$

and the $A_{b}$-eigenvalues take the simple form

$$
A_{\delta} R\left(a_{+}, a_{-}, b ; x, p\right)=2 \operatorname{ch}\left(\frac{\pi p}{a_{\delta}}\right) R\left(a_{+}, a_{-}, b ; x, p\right) .
$$

The latter properties are proved in Ref. 21 for the $b$-values occurring in (69). The interpolating function $R$ is detailed in Ref. 23; we study a more general function in Ref. 19 and elsewhere.

We conclude this contribution with some conjectures concerning $M$ particle eigenfunctions of the $M$ commuting $A \Delta \mathrm{Os}$. We believe that such joint eigenfunctions exist not only at the hyperbolic, but also at the elliptic level. (At the trigonometric level the joint eigenfunctions needed for quantum-mechanical purposes amount to the $A_{M-1}$ Macdonald polynomials, cf. Ref. 23, Scction 6.2.) In the hyperbolic case we expect self-duality (invariance under $\left(x_{1}, \ldots, x_{M}\right) \leftrightarrow\left(p_{1}, \ldots, p_{M}\right)$ ), as the quantum generalization of the classical self-duality first proved in Ref. 16. Moreover, the parameter symmetry (71) should still hold true for $M>2$, and the eigenvalues should be the obvious (scattering theory) generalizations of (72).

In the elliptic case we also expect the symmetry property (71). Note in this connection that $a_{+} \leftrightarrow a_{-}$symmetry would entail the joint eigenfunction property for the $M A \Delta \mathrm{O}$ s obtained by interchanging $a_{+}$and $a_{-}$; This second $A \Delta O$ family is readily seen to commute with the first one.

Finally, both at the elliptic and at the hyperbolic level we expect that the unitarity region is given by $b \in\left[0, a_{+}+a_{-}\right]$for all $M \geq 2$ and that the scattering (eigenfunction asymptotics) is factarized in terms of the (2-particle) $u$-function. To date, we are not aware of any evidence contradicting the above sccnario, but the evidence supporting it is mainly circumstantial.

\section{REFERENCES}

1. R. Askey and M. E. H. Ismail, A generalization of ultraspherical polynomials, Studies in Pure Mathematics (Boston, MA) (P. Erdös, ed.), Birkhäuser, 1983, pp. 55 78.

2. E. Billey, Algebraic nested Bethe ansatz for the elliptic Ruijsenaars model, math.qa/9806068.

3. J. Dittrich and V. I. Inozemtscv, On the structure of eigenvectors of the multidimensional Lamé operator, J. Phys. A 26 (1993), No. 16, L753-L756. 
4. G. Felder and A. Varchenko, Integral representation of solutions of the elliptic Knizhnak-Zamolodchikov-Bernand equations, Internat. Math. Res. Notices (1995), No. 5, 221-233.

5. Three formulas for eigenfunctions of integnable Sehrödinger operators, Compositio Math. 107 (1997), No. 2, 143-175.

6. Algebraic integrability of the two-body Ruigsenaars operator, Funct. Anal. Appl. 32 (1998), No. 2, 81-92.

7. G. Felder and A. N. Varchenko, Algebraic Bethe ansatz for the elliptic quanturn group $E_{\tau, \eta}\left(\mathrm{s}_{2}\right)$, Nucl. Phys. B 480 (1996), No. 1-2, 485-503, q-alg/9605024.

8. K. Hasegawa, Ruijsenaars' commuting difference operators as commutrng transfer matrices, Cornmun. Math. Phys. 187 (1997), No. 2, 289-325, q-alg/9512029.

9. V. I. Inozemtsev, Solution to three-magnon problem for $S=\frac{1}{2}$ periodic quantum spin chains with elliptic exchange, J. Math. Phys. 37 (1996), No. 1, 147-159.

10. Y. Komori, Notes on the elliptic Ruijsenaars operators, Lett. Math. Phys. 46 (1998), 147-155.

11. Y. Komori and K. Hikami, Elliptic $K$-matrix associated with Belawin's symmetric R-matrix, Nucl. Phys. B 494 (1997), 687-701.

12. I. M. Krichever and A. Zabrodin, Spin generalization of the Ruijsenaars-Schneider model, non-Abelian 20 Toda chain and representations of Sklyanin algebra, Russian Math. Surveys 50 (1995), No. 6, 1101-1150, hep-th/9505039.

13. E. H. Lieb and W. Liniger, Exact anahysis of an interacting Bose gas. 1. The general solution and the ground state, Phys. Rev. 130 (1963), $1605-1616$.

14. M. A. Olshanetsky and A. M. Perelomov, Classical integrable finitedimensional systems related to Lie algebras, Phys. Rep. 71 (1981), No. $5,313-400$.

15. Quantum integrable systems related to Lie algebras, Phys. Rep. 94 (1983), No. 6, 313-404.

16. S. N. M. Ruijsenaars, Action-angle maps and scattering theory for some firite-dimensional integrable systems. I. The pure soliton case, Commun. Math. Phys. 115 (1988), No. 1, 127-165. 
17. - Finite-dimensional soliton systerns, Integrable and Superintegrable Systems (B. A. Kupershmidt, ed.), World Scientific, Singapore, 1990, pp. 165-206.

18. __ First order analytic difference equations and integrable quantum systems, J. Math. Phys. 38 (1997), No. 2, 1069-1146.

19. ___ A generalized hypergeometric function satisfying four analytic difference equations of Askey-Wilson type, Commun. Math. Phys. 206 (1999), 639-690.

20. __ Generalized Lamé functions. I. The elliptic case, J. Math. Phys. 40 (1999), No. 3, 1595-1626.

21. __ Generalized Lamé functions. II. Hyperbolic and trigonometric specializations, J. Math. Phys. 40 (1999), No. 3, 1627-1663.

22. _ـ Relativistic Lamé functions: the special case $g=2, \mathrm{~J}$. Phys. A 32 (1999), 1737-1772.

23. Systems of Calogero-Moser type, Particles and Fields (Banff, 1994) (G. Semenoff and L. Vinet, eds.), CRM Series in Mathematical Physics, Springer, New York, 1999, pp. 251-352.

24. E. K. Sklyanin, Some algebraic structure connected with the Yang-Baxter equation, Funct. Anal. Appl. 16 (1982), No. 4, 263-270.

25. S__ Some algebraic structures connected with the Yang-Baxter equation. Representations of quantum algebras, Funct. Anal. Appl. 17 (1983), No. 4, 273-284.

26. E. T. Whittaker and G. N. Watson, A Course of Modern Analysis, Cambridge Univ, Press, Cambridge, 1927. 